\title{
Construction of locally plane graphs with many edges
}

\author{
Gábor Tardos* \\ Rényi Institute, Budapest, Hungary and \\ School of Computing Science, Simon Fraser University, Burnaby, BC \\ tardos@renyi.hu
}

\begin{abstract}
A graph drawn in the plane with straight-line edges is called a geometric graph. If no path of length at most $k$ in a geometric graph $G$ is self-intersecting we call $G k$-locally plane. The main result of this paper is a construction of $k$-locally plane graphs with a superlinear number of edges. For the proof we develop randomized thinning procedures for edge-colored bipartite (abstract) graphs that can be applied to other problems as well.
\end{abstract}

\section{Introduction}

A geometric graph $G$ is a straight-line drawing of a simple, finite (abstract) graph $(V, E)$, i.e., we identify the vertices $x \in V$ with distinct points in the Euclidean plane, and we identify any edge $\{x, y\} \in E$ with the straight line segments $x y$ in the plane. We assume that the edge $x y$ does not pass through any vertex of $G$ besides $x$ and $y$. We call $(V, E)$ the abstract graph underlying $G$. We say that the edges $e_{1}, e_{2} \in E$ cross if the corresponding line segments cross each other, i.e., if they have a common interior point. We say that a subgraph of $G$ is self-intersecting if it contains a pair of crossing edges.

Geometric graphs without crossing edges are plane drawings of planar graphs: they have at most $3 n-6$ edges if $n \geq 3$ is the number of vertices.

${ }^{*}$ Reserach partially supported by NSERC grant 329527 and by OTKA grants T-046234, AT048826 and NK-62321. 
Avital and Hanani [3], Erdös, and Perles initiated in the mid 1960s the systematic study of similar questions for more complicated forbidden configurations: Let $H$ be set of forbidden configurations (geometric subgraphs). What is the maximal number of edges of an $n$ vertex geometric graph not containing any configuration belonging to $H$ ? This problem can be regarded as a geometric version of the fundamental problem of extremal graph theory: What is the maximum number of edges that an abstract graph on $n$ vertices can have without containing subgraphs of a certain kind.

Many questions of the above type on geometric graphs have been addressed in recent years. In a number of papers linear upper bounds have been established for the number of edges of a graph, under various forbidden configurations. They include the configurations of three pairwise crossing edges [2, four pairwise crossing edges [1], the configurations of an edge crossed by many edges [9], or even two large stars with all edges of one of them crossing all edges of the other [13].

For a constant number of 5 or more pairwise crossing edges Pavel Valtr has the best result [11]: a geometric graph on $n$ vertices avoiding this configuration has $O(n \log n)$ edges but no construction is known with a super linear number of edges. Adam Marcus and the present author [4 building on an earlier result of Pinchasi and Radoičić [10] prove an $O\left(n^{3 / 2} \log n\right)$ bound on the number of edges of an $n$ vertex geometric graph not containing self-intersecting cycles of length four. No construction is known beating the $O\left(n^{3 / 2}\right)$ edges an abstract graph having no cycles of length four can have.

For surveys on geometric graph theory, consult [5], [6] and [8].

In this paper we consider forbidding self-intersecting paths. For $k \geq 3$ we call a geometric graph $k$-locally plane if it has no self-intersecting subgraph (whose underlying abstract graph is) isomorphic to a path of length at most $k$.

Pach et al. 7] consider 3-locally plane graphs, i.e., the case of geometric graphs with no self-intersecting paths of length three. They prove matching lower and upper bounds of $\Theta(n \log n)$ on the maximal number of edges of a 3 -locally plane graph on $n$ vertices.

We extend the the lower bound result of [7] by considering self-intersecting drawings of longer paths as forbidden configurations. Technically $k$-locally plane graphs are defined by forbidding self intersecting paths of length $k$ or shorter, but forbidding only self-intersecting paths of length exactly $k$ would lead to almost the same extremal function. Indeed, one can delete at most $n k$ edges from any graph on $n$ vertices, such that all the non-zero degrees in the remaining graph are larger than $k$. This ensures that all shorter paths can be extended to a path of length $k$. It is possible, but not likely, that 
if one only forbids paths of length $k$ with the first and last edges crossing significantly higher number of edges is achievable.

For even $k$ a geometric graph is $k$-locally plane if and only if the $k / 2$ neighborhood of any vertex $x$ is intersection free. Note that this requirement is much stronger than the similar condition on abstract graphs, namely that the $k / 2$ neighborhood of any point is planar. One can construct graphs with girth larger than $k$ and $\Omega\left(n^{\frac{k}{k-1}}\right)$ edges. In such a graph the $k / 2$ neighborhood of any vertex is a tree, still by [7] the graph does not even have 3-locally plane drawing.

Extending the lower bound result in [7] we prove in Theorem 3 that for arbitrary fixed $k \geq 3$ there exist $k$-locally plane graphs on $n$ vertices with $\Omega(n \log (\lfloor k / 2\rfloor) n)$ edges. Here $\log ^{(t)}$ denotes $t$ times iterated logarithm and the hidden constant in $\Omega$ depends on $k$. Given two arbitrarily small disks in the plane we can even ensure that all edges of the constructed graph connect a vertex from the first disk with another vertex from the second. This ensures that all the edges of the constructed geometric graph are arbitrarily close to each other in length and direction. In the view of the author this makes the existence of a high average degree (or for that matter high minimum degree) 100-locally plane graphs even more surprising.

As a simple corollary we can characterize the abstract graphs $H$ such that any geometric graph having no self-intersecting subgraph isomorphic to $H$ has a linear number of edges. These graphs $H$ are the forests with at least two nontrivial components. To see the linear bound for the number of edges of a geometric graph avoiding a self-intersecting copy of such a forest $H$ first delete a linear number of edges from an arbitrary geometric graph $G$ until all non-zero degrees of the remaining geometric graph $G^{\prime}$ are at least $|V(H)|$. If $G^{\prime}$ is crossing free the linear bound of the number of edges follows. If you find a pair of crossing edges in $G^{\prime}$ they can be extended to a subgraph of $G^{\prime}$ isomorphic to $H$. On the other hand, if $H$ contains a cycle, then even an abstract graph avoiding it can have a super-linear number of edges. If $H$ is a tree of diameter $k$, then a $k$-locally plane geometric graph has no self-intersecting copy of $H$. Notice that the extremal number of edges in this case (assuming $k>2$ ) is $O(n \log n)$ by [7, thus much smaller than the $\Omega\left(n^{\alpha}\right)$ edges $(\alpha>1)$ for forbidden cycles.

The main tool used in the proof of the above result is a randomized thinning procedure that takes a $d$ edge colored bipartite graph of average degree $\Theta(d)$ and returns a subgraph on the same vertex set with average degree $\Theta(\log d)$ that does not have a special type of colored path (walk) of length four. The procedure can be applied recursively to obtain a subgraph 
avoiding longer paths of certain types. We believe this thinning procedure to be of independent interest. In particular it can be used to obtain optimal 0-1 matrix constructions for certain avoided submatrix problems, see the exact statement in Section 4 and the details in [12].

In Section 2 we define two thinning procedures for edge colored bipartite graphs and prove their main properties. This is the most technical part of the paper. While these procedures proved useful in other setting too (and the author finds the involved combinatorics appealing) this entire section can be skipped if one reads the definition of $k$-flat graphs (the two paragraphs before Lemma 2.9) and is willing to accept Corollary 2.1 at the end of the section (we also use the simple observation in Lemma 2.10). In fact, in order to understand the main ideas behind the main result of this paper it is recommended to skip Section 2 on the first reading and to go straight to Section 3 where we use Corollary 2.1 to construct locally plane graphs with many edges. In Section 4 we comment on the optimality of the thinning procedures and have some concluding remarks.

\section{Thinning}

In this section we state and prove combinatorial statements about edge colored abstract graphs, i.e., we do not consider here geometric graphs at all. The connection to locally plane geometric graphs will be made clear in Section 3 ,

A bipartite graph is a triple $G=(A, B, E)$ with disjoint vertex sets $A$ and $B$ (called sides) and edge set $E \subseteq A \times B$. In particular, all graphs considered in this paper are simple, i.e., they do not have multiple edges or loops. The edge connecting the vertices $x$ and $y$ of $G$ is denoted by $x y$ or $(x, y)$. The latter notation is only used if $x \in A$ and $y \in B$. By a $d$ edge coloring of a graph we mean a mapping $\chi: E \rightarrow\{1,2, \ldots d\}$ such that adjacent edges receive different colors. When we do not specify $d$ we call such coloring a proper edge coloring but we always assume that the "set of colors" are linearly ordered. The degree of any vertex in $G$ is at most the number $d$ of colors, and our results are interesting if the average degree is close to $d$. Unless stated otherwise the subgraphs of an edge colored graph are considered with the inherited edge coloring. Our goal is to obtain a subgraph of $G$ with as many edges as possible without containing a certain type of colored path or walk. 


\subsection{Heavy paths}

A simple example of the above concept is the following. We call a path $P=v_{0} v_{1} v_{2} v_{3}$ of length 3 heavy if $v_{0} \in B$ and the colors $c_{1}=\chi\left(v_{0} v_{1}\right)$, $c_{2}=\chi\left(v_{1} v_{2}\right), c_{3}=\chi\left(v_{2} v_{3}\right)$, satisfy $c_{2}<c_{1} \leq c_{3}$. The next lemma describes a thinning procedure that gets rid of heavy paths. Although we do not need this lemma in our construction, we present it as a simple analogue of our results for more complicated forbidden walks.

Lemma 2.1. Let $G=(A, B, E)$ be a bipartite graph with a proper edge coloring $\chi: E \rightarrow\{1,2, \ldots d\}$. Then there exists a subgraph $G^{\prime}=\left(A, B, E^{\prime}\right)$ of $G$ with $\left|E^{\prime}\right| \geq|E| /(3\lceil\sqrt{d}\rceil)$ that does not contain heavy paths.

The constant 3 in the lemma could be replaced by the base of the natural logarithm. Notice that if $G$ had average degree $\Theta(d)$, then the average degree of $G^{\prime}$ is $\Omega(\sqrt{d})$.

Proof: Let $t=\lceil\sqrt{d}\rceil$ and select a uniform random value $i_{y} \in\{1,2, \ldots, t\}$ independently for each vertex $y \in B$. We say that an edge $e \in E$ is of class $\lceil\chi(e) / t\rceil$. We call an edge $e=(x, y) \in E$ eligible if its class is $i_{y}$. Let the subgraph $G^{\prime}=\left(A, B, E^{\prime}\right)$ consist of those eligible edges $e=(x, y) \in E$ for which there exists no other eligible edge $\left(x, y^{\prime}\right) \in E$ of the same class. Note that the words "class" and "eligible" will be used in a different meaning when defining the two thinning procedures in the next subsection.

By the construction, all edges incident to a vertex $x \in A$ have different classes and all edges incident to a vertex $y \in B$ have the same class. Let $e_{1}$, $e_{2}$ and $e_{3}$ form a path in $G^{\prime}$ starting in $B$. Then $e_{2}$ and $e_{3}$ are of the same class, while the class of $e_{1}$ is different. For the colors $c_{i}=\chi\left(e_{i}\right)$ this rules out the order $c_{2}<c_{1} \leq c_{3}$. Thus, $G^{\prime}$ does not contain a heavy path. Note that another order, $c_{3} \leq c_{1}<c_{2}$ is also impossible.

The number of edges in $G^{\prime}$ depends on the random choices we made. Any edge $(x, y) \in E$ is eligible with probability $1 / t$, and this is independent for all the edges incident to a vertex $x \in A$. As edges of a fixed color form a matching, there are at most $t$ edges of any given class incident to $x$. Thus, we have

$$
\operatorname{Pr}\left[(x, y) \in E^{\prime}\right] \geq \frac{(1-1 / t)^{t-1}}{t}>\frac{1}{3 t} .
$$

The expected number of edges in $G^{\prime}$ is

$$
\operatorname{Exp}\left[\left|E^{\prime}\right|\right] \geq \frac{|E|}{3 t}
$$

It is possible to choose the random variables $i_{y}$ so that the size of $E^{\prime}$ is at least as large as its expected value. This proves the lemma. 


\section{$2.2 \quad$ Fast and slow walks}

Next we turn to more complicated forbidden subgraphs. For motivation we mention that self-crossing paths of length 4 in the 3-locally plane graphs of [7] (considered with their natural edge coloring) are exactly the fast walks (to be defined below). For technical reasons, it will be more convenient to consider walks, i.e., to permit that a vertex is visited more than once, but we will not allow backtracking, i.e., turning back on the same edge immediately after it was traversed. Thus, for us a walk of length $k$ is a sequence $v_{0}, v_{1}, \ldots, v_{k}$ of vertices in the graph such that $v_{i-1} v_{i}$ is an edge for $1 \leq i \leq k$ and $v_{i-2} \neq v_{i}$ for $2 \leq i \leq k$. The $\chi$-coloring (or simply coloring) of this walk is the sequence $\left(\chi\left(v_{0} v_{1}\right), \chi\left(v_{1} v_{2}\right), \ldots, \chi\left(v_{k-1} v_{k}\right)\right)$ of the colors of the edges of the walk. If $\chi$ is a proper edge coloring, then any two consecutive elements of the coloring sequence are different.

We use log to denote the binary logarithm. We introduce the notation $P(a, b)$ for two non-equal strings $a, b \in\{0,1\}^{t}$ to denote the first position $i \in\{1,2, \ldots, t\}$, where $a$ and $b$ differ. We consider the set $\{0,1\}^{t}$ to be ordered lexicographically, i.e., for $a, b \in\{0,1\}^{t}$ we have $a<b$ if $a$ has 0 in position $P(a, b)$ (and thus $b$ has 1 there).

The following trivial observation is used often in this paper. We state it here without a proof.

Lemma 2.2. Let $t \geq 1$ and let $a, b$ and $c$ be distinct binary strings of length $t$ with $P(a, b)<P(a, c)$. We have $P(b, c)=P(a, b)$. Furthermore $a>b$ implies $c>b$, and $a<b$ implies $c<b$.

A walk of length 4 with coloring $\left(c_{1}, c_{2}, c_{3}, c_{4}\right)$ is called a fast walk if $c_{2}<c_{3}<c_{4} \leq c_{1}$. Note that a fast walk may start in either class $A$ or $B$. We call a walk of length 4 a slow walk if it starts in the class $B$ and its coloring $\left(c_{1}, c_{2}, c_{3}, c_{4}\right)$ satisfies $c_{2}<c_{3}<c_{4}$ and $c_{2}<c_{1} \leq c_{4}$. Note that either the color $c_{1}$ or $c_{3}$ can be larger in a slow walk, or they can be equal.

The two thinning procedures below find a random subgraph of an edgecolored bipartite graph. One is designed to avoid slow walks, the other is designed to avoid fast walks.

Lexicographic thinning Let $G=(A, B, E)$ be a bipartite graph and let $\chi: E \rightarrow\{1, \ldots, d\}$ be a proper edge coloring with $d \geq 2$. Lexicographic thinning is a randomized procedure that produces a subset $E^{\prime} \subseteq E$ of the edges and the corresponding subgraph $G^{\prime}=\left(A, B, E^{\prime}\right)$ of $G$ as follows:

Let $t=\left\lceil\frac{\log d}{2}\right\rceil+1$. Let $H$ be the set of triplets $(a, i, z)$, where $a \in\{0,1\}^{t}$, $i \in\{2,3, \ldots, t\}, z \in\left\{1,2,3, \ldots 2^{i}\right\}$, and $a$ has 0 in position $i$. Straightforward calculation gives that $|H|=2^{2 t}-2^{t+1} \geq 2 d$. 
We order $H$ lexicographically, i.e., $(a, i, z)<(b, j, s)$ if $a<b$, or $a=b$ and $i<j$, or $(a, i)=(b, j)$ and $z<s$.

Consider the following random function $F:\{1, \ldots, d\} \rightarrow H$. We select uniformly at random the value $F(1)=(a, i, z) \in H$ with the property that the first bit of $a$ is 0 . We make $F(2)$ to be the next element of $H$ larger than $F(1)$, and in general $F(k)$ is the next element of $H$ larger than $F(k-1)$ for $2 \leq k \leq d$. As $|H| \geq 2 d$ and $F(1)$ is chosen from the first half of $H$, this defines $F$. In what follows we simply identify the color $k$ with the element $F(k) \in H$ without any reference to the function $F$.

We say that $(a, i, z) \in H$ and any edge with this color is of class $a$ and type $i$, while $z$ will play no role except in counting how many values it can take.

We choose an independent uniform random value $a_{x} \in\{0,1\}^{t}$ for each vertex $x \in A \cup B$. Let $e=(x, y) \in E$ be an edge of class $a$ and type $i$. We say that $e$ is eligible if $a=a_{y}<a_{x}$ and $P\left(a, a_{x}\right)=i$. Let the subgraph $G^{\prime}=\left(A, B, E^{\prime}\right)$ contain those edges $e \in E$ that are eligible but not adjacent to another eligible edge $e^{\prime}$ of the same type as $e$.

Reversed thinning Let $G=(A, B, E)$ be a bipartite graph and let $\chi$ : $E \rightarrow\{1, \ldots, d\}$ be a proper edge coloring with $d \geq 2$. Reversed thinning is a randomized procedure that produces a subset $E^{\prime} \subseteq E$ of the edges and the corresponding subgraph $G^{\prime}=\left(A, B, E^{\prime}\right)$ of $G$.

Reversed thinning is almost identical to the lexicographic thinning, the only difference is in the ordering of the set $H$. We define $t$ and $H$ as in the case of lexicographic thinning. Recall that $H$ is the set of triplets $(a, i, z)$ where $a \in\{0,1\}^{t}, i \in\{2,3, \ldots, t\}, z \in\left\{1,2, \ldots 2^{i}\right\}$, and $a$ has 0 in position $i$. We still order $\{0,1\}^{t}$ lexicographically, but now we reverse the lexicographic order of $H$ in the middle term $i$. That is, we have $(a, i, z)<(b, j, s)$ if $a<b$, or $a=b$ and $i>j$ or $(a, i)=(b, j)$ and $z<s$.

We define the function $F:\{1, \ldots, d\} \rightarrow H$, the types and classes of colors and edges, eligible edges and the subset $E^{\prime}$ of edges the same way as for the lexicographic thinning, but using this modified ordering of $H$.

Note that we associated a type in $\{2, \ldots, t\}$ and a class in $\{0,1\}^{t}$ to each edge in either procedure and they satisfy that

- an edge with a smaller class has smaller color;

- among edges of equal class an edge with smaller type has smaller color in the case of lexicographic thinning and it has larger color in the case of reversed thinning; 
- among the edges incident to a vertex at most $2^{i}$ have the same class $a$ and the same type $i$.

Proving most of the properties of the thinning procedures this is all we need to know about how classes and types are associated to the edges and we could use a deterministic scheme for $F$. But for Lemma 2.6 we need that all types are well represented and the randomization in the identification function $F$ (as well as the dummy first bit of the class) is introduced to ensure this on the average. This randomization is not needed if one assumes all color classes have roughly the same size.

The next lemmas state the basic properties of the thinning procedure. Lemma 2.3 lists common properties of the two procedures, while Lemmas 2.4 and 2.5 state the result of the thinning satisfies its "design criteria" avoiding slow or fast walks. Finally Lemma 2.6 shows that enough edges remain in the constructed subgraphs on average. Note that Lemmas 2.4 and 2.5] are special cases of the more complex Lemmas 2.7 and 2.8 proved independently. We state and prove the simple cases separately for clarity, but these proofs could be skipped.

Lemma 2.3. Let $G=(A, B, E)$ be a bipartite graph with a proper edge coloring $\chi: E \rightarrow\{1, \ldots, d\}$. If $G^{\prime}=\left(A, B, E^{\prime}\right)$ is the result of either the lexicographic or the reversed thinning then we have

a) Adjacent edges in $G^{\prime}$ have distinct types.

b) If two edges of $G^{\prime}$ meet in $B$, they have the same class.

c) Suppose two distinct edges e and $e^{\prime}$ of $G^{\prime}$ meet in $A$. Let their classes and types be $a, a^{\prime}$ and $i, i^{\prime}$, respectively. If $i<i^{\prime}$ then $a<a^{\prime}$ and $P\left(a, a^{\prime}\right)=i$.

d) G' has no heavy path.

Proof: The definition of $E^{\prime}$ immediately gives a).

For b) note that all eligible edges incident to $y \in B$ have $a_{y}$ for class.

For c) let $x \in A$ be the common vertex of the two edges and apply Lemma 2.2 for $a_{x}, a$, and $a^{\prime}$.

Finally d) follows since if a walk of $G^{\prime}$ starts in $B$ then its coloring $\left(c_{1}, c_{2}, c_{3}\right)$ must satisfy that $c_{1}$ and $c_{2}$ have different class by c) but $c_{2}$ and $c_{3}$ have the same class by $\mathrm{b}$ ), so $c_{2}<c_{1} \leq c_{3}$ is impossible.

Lemma 2.4. Let $G=(A, B, E)$ be a bipartite graph with a proper edge coloring. Lexicographic thinning produces a subgraph $G^{\prime}$ with no slow walk. 
Proof: Suppose $v_{0} v_{1} v_{2} v_{3} v_{4}$ is a walk in $G^{\prime}$ starting at $v_{0} \in B$ and let its coloring be $\left(c_{1}, c_{2}, c_{3}, c_{4}\right)$. Assume $c_{1}>c_{2}<c_{3}<c_{4}$. We show that $c_{1}>c_{4}$, so this walk is not slow. By Lemma $2.3 / \mathrm{a}, \mathrm{b}$, as $c_{2}$ and $c_{3}$ are colors of edges incident to $v_{2} \in B$, they have the same class, but they have different types: $c_{2}=(a, i, z), c_{3}=(a, j, s)$ with $i \neq j$. We use lexicographic ordering, so $c_{2}<c_{3}$ implies $i<j$. Both $c_{1}$ and $c_{2}$ are colors of edges incident to $v_{1} \in A$, so by Lemma 2.3/c their classes are different. Since $c_{1}>c_{2}$ we have $b>a$ for the class $b$ of $c_{1}$. Still by Lemma 2.3/ c $P(a, b)=i$. Similarly, $c_{3}$ and $c_{4}$ are colors of distinct edges in $E^{\prime}$ incident to $v_{3} \in A$, so they have different classes. As $c_{3}<c_{4}$ we have $c>a$ for the class $c$ of $c_{4}$. We have $P(a, c)=j$. By Lemma 2.2 we have $b>c$. This proves $c_{1}>c_{4}$ as claimed.

Lemma 2.5. Let $G=(A, B, E)$ be a bipartite graph with a proper edge coloring. Reversed thinning produces a subgraph $G^{\prime}$ with no fast walk.

Proof: Suppose $v_{0} v_{1} v_{2} v_{3} v_{4}$ is a walk in $G^{\prime}$ with coloring $\left(c_{1}, c_{2}, c_{3}, c_{4}\right)$. Assume $c_{1}>c_{2}<c_{3}<c_{4}$. We show that $c_{1}<c_{4}$, so this walk is not fast. First assume the walk starts at $v_{0} \in A$. As $G^{\prime}$ does not contain a heavy path, $v_{3} v_{2} v_{1} v_{0}$ is not heavy, so $c_{1}<c_{3}$. This implies $c_{1}<c_{4}$ as claimed.

Now assume $v_{0} \in B$. Just as in the proof of the previous lemma, $c_{2}$ and $c_{3}$ are colors of edges incident to $v_{2} \in B$, so they have the same class, but they have different types: $c_{2}=(a, i, z), c_{3}=(a, j, s)$ with $i \neq j$. We use the reversed ordering, so $c_{2}<c_{3}$ implies $i>j$. Both $c_{1}$ and $c_{2}$ are colors of edges incident to $v_{1} \in A$, so their classes are different. Since $c_{1}>c_{2}$ we have $b>a$ for the class $b$ of $c_{1}$ and $P(a, b)=i$. Similarly, $c_{3}$ and $c_{4}$ are colors of distinct edges in $E^{\prime}$ incident to $v_{3} \in A$, so they have different classes. As $c_{3}<c_{4}$ we have $c>a$ for the class $c$ of $c_{4}$ and $P(a, c)=j$. By Lemma 2.2 we have $c>b$. This proves $c_{1}<c_{4}$ as claimed.

Below we estimate the number of edges in $E^{\prime}$. Recall that both thinning procedures are randomized. We can show that the subgraphs they produce have a large expected number of edges. We did not make any effort to optimize for the constant in this lemma.

Lemma 2.6. Let $G=(A, B, E)$ be a bipartite graph with a d edge coloring. Let $G^{\prime}=\left(A, B, E^{\prime}\right)$ be the result of either the lexicographic or the reversed thinning. We have

$$
\operatorname{Exp}\left[\left|E^{\prime}\right|\right] \geq \frac{t-1}{240 d}|E| \geq \frac{\log d}{480 d}|E| .
$$

Proof: We compute the probability for a fixed edge $e=(x, y) \in E$ to end up in $E^{\prime}$. For this we break down the random process producing $E^{\prime}$ 
into three phases. In the first phase we select $F$. With $F$ the color $\chi(e)$ is identified with an element of $H$, most importantly, the type of $e$ is fixed. In the second phase we select $a_{x}$ and $a_{y}$ uniformly at random. These choices determine if $e$ is eligible. If $e$ is not eligible then $e \notin E^{\prime}$. So in the third phase we consider $F, a_{x}$, and $a_{y}$ fixed and assume $e$ is eligible. We select the random values $a_{z}$ for vertices $z \neq x, y$. This effects if other edges are eligible and if $e \in E^{\prime}$. Here is the detailed calculation:

Let $e \in E$ have the color $\chi(e)=k \in\{1,2, \ldots, d\}$. The choice of $F$ in the first phase determines $F(k)=(a, i, z) \in H$. By the construction of $F$, if we call $a^{\prime}$ the last $t-1$ bits of $a$ then $\left(a^{\prime}, i, z\right)$ is uniformly distributed among all its possible values. In particular, the probability that $e$ becomes a type $i$ edge is exactly

$$
\operatorname{Pr}[e \text { is of type } i]=\frac{2^{t+i-2}}{2^{2 t-1}-2^{t}}=\frac{2^{i}}{2^{t+1}-4} .
$$

For phase two we consider the function $F$ identifying colors with elements of $H$ fixed. Consider an edge $e=(x, y)$ of color $(a, i, z) \in H$. This edge is eligible if $a_{y}=a<a_{x}$ and $P\left(a, a_{x}\right)=i$. This determines $a_{y}$ and the first $i$ bits of $a_{x}$. Recall that by the definition of $H$ the string $a$ has 0 in position $i$. Thus, the probability that the edge $e$ of type $i$ is eligible is exactly $2^{-t-i}$.

Assume for the third phase that $e$ is eligible. Consider another edge $e^{\prime}=\left(x, y^{\prime}\right) \in E$ with color $\chi\left(e^{\prime}\right)=\left(a^{\prime}, i, z^{\prime}\right)$ of type $i$. If $a$ and $a^{\prime}$ do not agree in the first $i$ positions, then $e^{\prime}$ is not eligible. If they agree in the first $i$ positions, then $e^{\prime}$ is eligible if and only if $a_{y^{\prime}}=a^{\prime}$, so with probability $2^{-t}$. Let $k_{e}^{\prime}$ be the number of edges $\left(x, y^{\prime}\right)$ of type $i$ with the first $i$ digits of their class agreeing with $a$, but not counting $e$ itself. We have $k_{e}^{\prime}<2^{t}$.

Consider now an edge $e^{\prime \prime}=\left(x^{\prime \prime}, y\right) \in E$ with color $\left(a^{\prime \prime}, i, z^{\prime \prime}\right)$ such that $e^{\prime \prime} \neq e$. As $e$ is eligible, $e^{\prime \prime}$ can only be eligible if $a^{\prime \prime}=a$. If $a^{\prime \prime}=a$ then $e^{\prime \prime}$ is eligible if and only if $a_{x}$ and $a_{x^{\prime \prime}}$ agree in the first $i$ digits. This happens with probability $2^{-i}$. For the number $k_{e}^{\prime \prime}$ of the edges $\left(x^{\prime \prime}, y\right) \neq e$ of type $i$ and class $a$ we have $k_{e}^{\prime \prime}<2^{i}$.

In phase three the eligibility of all these edges $e^{\prime}$ and $e^{\prime \prime}$ adjacent with $e$ are independent events.

Still consider the function $F$ fixed. The total probability for an edge $e$ of type $i$ to be in $E^{\prime}$ is

$$
\begin{aligned}
\operatorname{Pr}\left[e \in E^{\prime} \mid F\right] & =2^{-t-i}\left(1-2^{-t}\right)^{k_{e}^{\prime}}\left(1-2^{-i}\right)^{k_{e}^{\prime \prime}} \\
& \geq 2^{-t-i}\left(1-2^{-t}\right)^{2^{t}-1}\left(1-2^{-i}\right)^{2^{i}-1} \\
& >2^{-t-i} / 7.5 .
\end{aligned}
$$


The total probability of $e \in E^{\prime}$ can be calculated from the distribution of its type and the above conditional probability depending on its type:

$$
\operatorname{Pr}\left[e \in E^{\prime}\right]>\sum_{i=2}^{t} \frac{2^{i}}{2^{t+1}-4} \cdot \frac{2^{-t-i}}{7.5}>\frac{t-1}{15 \cdot 2^{2 t}}>\frac{t-1}{240 d} .
$$

The expected number of edges in $E^{\prime}$ is then

$$
\operatorname{Exp}\left[\left|E^{\prime}\right|\right]>\frac{t-1}{240 d}|E| \geq \frac{\log d}{480 d}|E| .
$$

Theorem 1. Let $G=(A, B, E)$ be a bipartite graph with a proper edge coloring. There exists a subgraph $G^{\prime}=\left(A, B, E^{\prime}\right)$ of $G$ without a slow walk and with $\left|E^{\prime}\right|>\frac{\log d}{480 d} \cdot|E|$. Similarly, there exists a subgraph $G^{\prime \prime}=\left(A, B, E^{\prime \prime}\right)$ of $G$ without a fast walk and with $\left|E^{\prime \prime}\right|>\frac{\log d}{480 d} \cdot|E|$.

Proof: By Lemmas 2.4 and 2.5 the results of the lexicographic and reversed thinnings avoid the slow and fast walks, respectively. There exists an instance of the random choices with the size of $E^{\prime}$ being at least its expectation given in Lemma 2.6. This proves the theorem.

\subsection{Longer forbidden walks}

Here we generalize the concept of fast and slow walks to longer walks. Consider a bipartite graph $G=(A, B, E)$ with a proper edge coloring. For $k \geq 2$ we call a walk of length $2 k$ in $G$ a $k$-fast walk if its coloring $\left(c_{1}, \ldots, c_{2 k}\right)$ satisfies $c_{1}>c_{2}>\ldots>c_{k}<c_{k+1}<c_{k+2}<\ldots<c_{2 k}$ and $c_{1} \geq c_{2 k}$. For $k \geq 2$ we call a walk of length $2 k$ in $G$ a $k$-slow walk if its coloring $\left(c_{1}, \ldots, c_{2 k}\right)$ satisfies the following: $c_{2 j-1}>c_{2 j}$ for $1 \leq j \leq k / 2 ; c_{2 j}<c_{2 j+1}$ for $1 \leq j<k / 2$; $c_{2 j-1}<c_{2 j}$ for $k / 2<j \leq k ; c_{2 j}>c_{2 j+1}$ for $k / 2 \leq j<k$; and finally $c_{1} \geq c_{2 k}$. If a $k$-slow walk starts in the vertex set $B$ we call it a $(k, B)$-slow walk, otherwise it is a $(k, A)$-slow walk.

Notice that 2 -fast walks are the fast walks and $(2, B)$-slow walks are the slow walks with their orientation reversed. For the coloring $\left(c_{1}, \ldots, c_{2 k}\right)$ of a $k$-fast walk $c_{j}$ is in between $c_{j-1}$ and $c_{j+1}$ for all $1<j<2 k, j \neq k$, while $c_{k}$ is the smallest color on this list. For the coloring $\left(c_{1}, \ldots, c_{2 k}\right)$ of a $k$-slow walk the situation is reversed: the only index $1<j<2 k$ with $c_{j}$ being in between $c_{j-1}$ and $c_{j+1}$ is the index $j=k$.

In order to apply the lexicographic and reversed thinning recursively we have to change the coloring of the subgraph. Let $G=(A, B, E)$ be a 
bipartite graph with a proper edge coloring given by $\chi: E \rightarrow\{1, \ldots, d\}$. Let $G^{\prime}=\left(A, B, E^{\prime}\right)$ the result of the lexicographic or the reversed thinning of $G$. Recall that the edges in $E^{\prime}$ have a type $2 \leq i \leq t$ with $t=\lceil(\log d) / 2\rceil+1$. The type edge coloring of $G^{\prime}$ is the map $\chi^{\prime}: E^{\prime} \rightarrow\{1, \ldots, t-1\}$ defined by $\chi^{\prime}(e)=t+1-i$ for an edge $e \in E^{\prime}$ of type $i$. By Lemma 2.3/a $\chi^{\prime}$ is a proper edge coloring of $G^{\prime}$.

Lemma 2.7. Let $G=(A, B, E)$ be a bipartite graph with proper edge coloring given by $\chi: E \rightarrow\{1, \ldots, d\}$. Let $G^{\prime}=\left(A, B, E^{\prime}\right)$ the result of the lexicographic thinning of $G$. Let $\chi^{\prime}$ be the type edge coloring of $G^{\prime}$ and let $k \geq 2$. If a subgraph $G^{\prime \prime}=\left(A, B, E^{\prime \prime}\right)$ of $G^{\prime}$ with its edge coloring given by $\chi^{\prime}$ has no $\left(k^{\prime}, A\right)$-slow walk for $2 \leq k^{\prime}<k$ then $G^{\prime \prime}$ with its edge coloring given by $\chi$ has no $(k, B)$-slow walk.

Notice that the $k=2$ case of this lemma gives a second proof of Lemma 2.4.

Proof: Let $W=v_{0} v_{1} \ldots v_{2 k}$ be a walk in $G^{\prime \prime}$ starting at $v_{0} \in B$ and let its $\chi$-coloring be $\left(c_{1}, c_{2}, \ldots, c_{2 k}\right)$. Assume that $c_{i}>c_{i+1}$ or $c_{i}<c_{i+1}$ for $1 \leq i<2 k$ as required in the definition of a $(k, B)$-slow walk. We need to show $c_{1}<c_{2 k}$.

We identify the colors of $\chi$ with the triplets $(a, i, z) \in H$ as in the definition of lexicographic thinning. We let $c_{j}=\left(a_{j}, i_{j}, z_{j}\right)$. The $\chi^{\prime}$-coloring of $W$ is $\left(t+1-i_{1}, \ldots, t+1-i_{2 k}\right)$. We have $i_{j} \neq i_{j+1}$ for $1 \leq j<2 k$.

For $1 \leq j<k$ the colors $c_{2 j}$ and $c_{2 j+1}$ are colors of distinct edges incident to $v_{2 j} \in B$, so by Lemma 2.3/b their class is the same: $a_{2 j}=a_{2 j+1}$. We consider lexicographic thinning, so the order between $c_{2 j}$ and $c_{2 j+1}$ is the same as the order between their types: $i_{2 j}$ and $i_{2 j+1}$. For $1 \leq j<k / 2$ we have $i_{2 j}<i_{2 j+1}$ but for $k / 2 \leq j<k$ we have $i_{2 j}>i_{2 j+1}$.

For $1 \leq j \leq k$ the colors $c_{2 j-1}$ and $c_{2 j}$ are colors of edges incident to $v_{2 j-1} \in A$. By Lemma 2.3/c the classes of these colors do not agree, and the ordering between the classes, between the types, and between the colors themselves are the same. Thus, for $1 \leq j \leq k / 2$ we have $a_{2 j-1}>a_{2 j}$ and $i_{2 j-1}>i_{2 j}$. For $k / 2<j \leq k$ we have $a_{2 j-1}<a_{2 j}$ and $i_{2 j-1}<i_{2 j}$. Also by Lemma 2.3/c for all $1 \leq j \leq k$ we have $P\left(a_{2 j-1}, a_{2 j}\right)=\min \left(i_{2 j-1}, i_{2 j}\right)$.

The sequence $a_{1}, a_{2}, \ldots, a_{k}$ is monotone decreasing and it changes only in every other step. The first positions of change between distinct consecutive elements are $i_{2}, i_{4}, \ldots, i_{2\lfloor k / 2\rfloor}$. So we have $a_{1}>a_{k}$ and $P\left(a_{1}, a_{k}\right)=\min \left(S_{1}\right)$ for the set $S_{1}=\left\{i_{2}, i_{4}, \ldots, i_{2\lfloor k / 2\rfloor}\right\}$.

Similarly, $a_{k}, a_{k+1}, \ldots, a_{2 k}$ is monotone increasing and it changes only in every other step. The first positions of change between distinct con- 
secutive elements are $i_{2\lfloor k / 2\rfloor+1}, \ldots, i_{2 k-3}, i_{2 k-1}$. So we have $a_{k}<a_{2 k}$ and $P\left(a_{k}, a_{2 k}\right)=\min \left(S_{2}\right)$ for the set $S_{2}=\left\{i_{2\lfloor k / 2\rfloor+1}, \ldots, i_{2 k-3}, i_{2 k-1}\right\}$.

Let us consider an arbitrary value $1 \leq j<k / 2$ and let $2 \leq k^{\prime}=k-$ $2 j+1<k$. Consider the $2 k^{\prime}$ long middle portion $W^{\prime}$ of the walk $W$ : let $W^{\prime}=v_{2 j-1} v_{2 j} \ldots v_{2 k-2 j+1}$. This is a walk of length $2 k^{\prime}$ in $G^{\prime \prime}$ starting at $v_{2 j-1} \in A$. By our assumption on $G^{\prime \prime}$ this is not a $\left(k^{\prime}, A\right)$-slow walk if considered with the coloring $\chi^{\prime}$. But the $\chi^{\prime}$-coloring of $W^{\prime}$ is $(t+1-$ $\left.i_{2 j}, t+1-i_{2 j+1}, \ldots, t+1-i_{2 k-2 j+1}\right)$ and the consecutive values in this list compare as required for a $\left(k^{\prime}, A\right)$-slow walk. Therefore, we must have $t+1-i_{2 j}<t+1-i_{2 k-2 j+1}$.

We have just proved $i_{2 j}>i_{2 k-2 j+1}$ for $1 \leq j<k / 2$. For even $k$ and $j=k / 2$ the same formula compares the types of two consecutive edges of $W$ and we have already seen its validity in that case too. For every element of the set $S_{1}$ we have just found a smaller element of the set $S_{2}$. Therefore, $\min \left(S_{1}\right)>\min \left(S_{2}\right)$. Using that $a_{2 k}>a_{k}$ and $P\left(a_{1}, a_{k}\right)=\min \left(S_{1}\right)>$ $\min \left(S_{2}\right)=P\left(a_{k}, a_{2 k}\right)$ Lemma 2.2 gives $a_{1}<a_{2 k}$. This implies $c_{1}<c_{2 k}$ and finishes the proof of the lemma.

Lemma 2.8. Let $G=(A, B, E)$ be a bipartite graph with proper edge coloring given by $\chi: E \rightarrow\{1, \ldots, d\}$. Let $G^{\prime}=\left(A, B, E^{\prime}\right)$ the result of the reversed thinning of $G$. Let $\chi^{\prime}$ be the type edge coloring of $G^{\prime}$ and let $k \geq 2$. If a subgraph $G^{\prime \prime}=\left(A, B, E^{\prime \prime}\right)$ of $G^{\prime}$ with its edge coloring given by $\chi^{\prime}$ has no $\left(k^{\prime}, A\right)$-slow walk for $2 \leq k^{\prime}<k$ then $G^{\prime \prime}$ with its edge coloring given by $\chi$ has no $k$-fast walk.

Notice that the $k=2$ case of this lemma gives a second proof of Lemma 2.5,

Proof: The proof of this lemma is very similar to that of Lemma 2.7

Let $W=v_{0} v_{1} \ldots v_{2 k}$ be a walk in $G^{\prime \prime}$ and let its $\chi$-coloring be $\left(c_{1}, c_{2}, \ldots, c_{2 k}\right)$. Assume that $c_{1}>c_{2}>\ldots>c_{k}<c_{k+1}<c_{k+2}<\ldots<c_{2 k}$ as required in the definition of a $k$-fast walk. We need to show $c_{1}<c_{2 k}$. Instead, we prove the slightly stronger statement that the class of $c_{1}$ is smaller than the class of $c_{2 k}$. We first do that for walks starting in $B$ : assume that $v_{0} \in B$.

We identify the colors of $\chi$ with the triplets $(a, i, z) \in H$ as in the definition of reverse thinning. We let $c_{j}=\left(a_{j}, i_{j}, z_{j}\right)$. Note that the $\chi^{\prime}-$ coloring of $W$ is $\left(t+1-i_{1}, \ldots, t+1-i_{2 k}\right)$. We have $i_{j} \neq i_{j+1}$ for $1 \leq j<2 k$.

For $1 \leq j<k$ the colors $c_{2 j}$ and $c_{2 j+1}$ are colors of distinct edges incident to $v_{2 j} \in B$, so by Lemma 2.3/b their classes are the same: $a_{2 j}=a_{2 j+1}$. Thus the order between $c_{2 j}$ and $c_{2 j+1}$ is determined by the order between $i_{2 j}$ and $i_{2 j+1}$, but as we use the reversed ordering in $H$ the order between $c_{2 j}$ and 
$c_{2 j+1}$ is reversed compared to the order between $i_{2 j}$ and $i_{2 j+1}$. Specifically, for $1 \leq j<k / 2$ we have $i_{2 j}<i_{2 j+1}$ and for $k / 2 \leq j<k$ we have $i_{2 j}>i_{2 j+1}$. For $1 \leq j \leq k$ the colors $c_{2 j-1}$ and $c_{2 j}$ are colors of edges incident to $v_{2 j-1} \in A$. By Lemma 2.3/c the classes of these colors do not agree, and the ordering between the classes, between the types, and between the colors themselves are the same. Thus, for $1 \leq j \leq k / 2$ we have $a_{2 j-1}>a_{2 j}$ and $i_{2 j-1}>i_{2 j}$. For $k / 2<j \leq k$ we have $a_{2 j-1}<a_{2 j}$ and $i_{2 j-1}<i_{2 j}$. Also by Lemma 2.3 / c for $1 \leq j \leq k$ we have $P\left(a_{2 j-1}, a_{2 j}\right)=\min \left(i_{2 j-1}, i_{2 j}\right)$.

At this point we have the same ordering of the classes and types of the coloring of $W$ as in the proof of Lemma 2.7. We also have the same assumption that $G^{\prime \prime}$ with the edge coloring $\chi^{\prime}$ has no $\left(k^{\prime}, A\right)$-slow walk for $2 \leq k^{\prime}<k$. So we arrive to the same conclusion $a_{1}<a_{2 k}$ with an identical proof.

We finish the proof of the lemma by considering the alternative case when $W$ starts in $A$. Now $c_{1}$ and $c_{2}$ are colors of edges sharing a vertex $v_{1} \in B$, so by Lemma $2.3 / \mathrm{b}$ their classes are equal. Similarly, the classes of $c_{2 k-1}$ and $c_{2 k}$ are equal, so it is enough to prove that the class of $c_{2}$ is smaller than the class of $c_{2 k-1}$. For $k=2$ this follows directly from Lemma 2.3/c. For $k>2$ the walk $W^{\prime}=v_{1} v_{2} \ldots v_{2 k-1}$ is exactly the type of walk we considered for $k_{0}=k-1$. As it starts in $B$ we have already proved that the class of its first edge is smaller than the class of its last edge. This finishes the proof of the case of a walk starting in $A$ and also the proof of Lemma 2.8 .

Lemmas 2.7 and 2.8 set the stage to use the thinning procedures recursively to get subgraphs avoiding $(k, B)$-slow or $k$-fast walks. In a single application of either thinning procedure the number $d$ of colors in the original coloring is replaced by $t-1=\lceil\log d / 2\rceil$ colors in the type coloring. Here $4(t-1)>\log (4 d)$, so after $k$ recursive calls we still have more than $\log ^{(k)}(4 d) / 4$ colors, where $\log ^{(k)}$ stands for the $k$ times iterated log functions. (Of course, this only makes sense if $\log ^{(k)}(4 d)>2$. Otherwise we can get stuck, as neither thinning procedure is defined in the pathetic case of $d=1$ colors.) Making optimal random choices we may assume that each thinning procedure yields at least the expected number of edges. Thus, the ratio of the number of edges and the number of colors decreases by at most a factor of 240 in each iteration. Clearly, the only interesting case is when the original average degree was $\Theta(d)$ in which case the average degree after $k$ iterations remains $\Theta\left(\log ^{(k)} d\right)$. The constant of proportionality depends on $k$.

Theorem 2. Let $G=(A, B, E)$ be a bipartite graph with a d edge coloring 
and let $k \geq 2$. There exists a subgraph $G^{\prime}=\left(A, B, E^{\prime}\right)$ of $G$ without a $\left(k^{\prime}, B\right)$-slow walk for any $2 \leq k^{\prime} \leq k$ and with $\left|E^{\prime}\right|>\frac{\log ^{(k-1)} d}{4 \cdot 240^{k-1} d}|E|$. Similarly, there exists a subgraph $G^{\prime \prime}=\left(A, B, E^{\prime \prime}\right)$ of $G$ without a $k^{\prime}$-fast walk for any $2 \leq k^{\prime} \leq k$ and with $\left|E^{\prime \prime}\right|>\frac{\log ^{(k-1)} d}{4 \cdot 240^{k-1} d}|E|$.

Proof: We apply the thinning procedures recursively. First we use lexicographic and reversed thinning to obtain subgraphs $G_{1}$ and $G_{2}$ of $G$, respectively. We make sure these graphs have at least as many edges as the expected number given in Lemma 2.6. If $k=2$ we are done, $G^{\prime}=G_{1}$ and $G^{\prime \prime}=G_{2}$ satisfy the conditions of the theorem. Otherwise we consider $G_{1}$ and $G_{2}$ with the type edge coloring. We find recursively their subgraphs $G^{\prime}$ and $G^{\prime \prime}$, respectively, avoiding $\left(k^{\prime}, A\right)$-slow walks for $2 \leq k^{\prime} \leq k-1$. This can be done because the sides $A$ and $B$ play symmetric roles. Finally, we apply Lemmas 2.7 and Lemma 2.8 to see that the subgraphs $G^{\prime}$ and $G^{\prime \prime}$, if considered with the original edge coloring of $G$, avoid all walks required in the theorem. The number of edges guaranteed in the subgraphs is calculated in the paragraph preceding the theorem and is at least the stated bound.

\section{$2.4 \quad k$-flat graphs}

In this subsection we establish that removing a linear number of edges from a $k$-fast walk free graph the resulting graph has special structural properties. We note here that the recursive thinning construction that we used to arrive at $k$-fast walk free graphs results in a graph that itself is $k$-flat as defined below. We chose however to keep the inductive part of the proof simple and concentrated only on $(k, B)$-slow and $k$-fast walks. We derive the more complicated properties from these simpler ones. Note that in this subsection we do not use that our graphs are bipartite.

Let $G$ be a graph and $\chi$ a proper edge coloring of $G$. We define the shaving of the graph $G$ to be the subgraph obtained from $G$ by deleting the edge with the largesr color incident to every (non-isolated) vertex. Clearly, we delete at most $n$ edges, where $n$ is the number of vertices in $G$. We define the $k$-shaving of $G$ to be the subgraph obtained from $G$ by repeating the shaving operation $k$ times. Clearly, we delete at most $k n$ edges for a $k$-shaving.

Let $W$ be a walk of length $m$ in a properly edge colored graph $G$, and assume its coloring is $\left(c_{1}, \ldots, c_{m}\right)$. We define the height function $h_{W}$ from 
$\{1, \ldots, m\}$ to the integers recursively letting $h_{W}(1)=0$ and

$$
h_{W}(i+1)=\left\{\begin{array}{lll}
h_{W}(i)+1 & \text { if } & c_{i+1}>c_{i} \\
h_{W}(i)-1 & \text { if } & c_{i+1}<c_{i}
\end{array}\right.
$$

for $1 \leq i<m$. Note $h_{W}(i)+i$ is always odd. This function considers how the colors of the edges in the walk change, in particular, how many times the next color is larger and how many times it is smaller than the previous color.

We call a graph $G$ with proper edge coloring $k$-flat if the following is true for every walk $W$ in $G$. Let $m \geq 2$ be the length of $W$, let $\left(c_{1}, \ldots, c_{m}\right)$ be the coloring of $W$ and assume that the height function satisfies $h_{W}(i)<0$ for $2 \leq i \leq m$. If $m \leq 2 k+1$ or $h_{W}(i) \geq-k$ for all $i$ then $c_{1}>c_{m}$.

Lemma 2.9. Let $G$ be properly edge colored graph. Let $k \geq 1$ and assume $G$ has no $k^{\prime}$-fast walk for $2 \leq k^{\prime} \leq k$. Then the $(k-1)$-shaving $G^{\prime}$ of $G$ is $k$-flat.

Proof: We prove the following slightly stronger statement by induction on $m$. Let $W=v_{0} \ldots v_{m}$ be a walk of length $m$ in $G$ with coloring $\left(c_{1}, \ldots, c_{m}\right)$. Let $1 \leq j \leq m$ be the largest index such that $h_{W}(j)=1-j$. Assume the walk $v_{j} v_{j+1} \ldots v_{m}$ is in the $(k-1)$-shaving $G^{\prime}$ of $G$. Also assume that $h_{W}(i)<0$ for $2 \leq i \leq m$. If $m \leq 2 k+1$ or $h_{W}(i) \geq-k$ for all $i$ then we claim $c_{1}>c_{m}$. This statement is stronger than Lemma 2.9 since it allows for the initial decreasing segment of $W$ be outside $G^{\prime}$.

If $j=m$ the statement of the claim is obvious from the definition of the height function. This covers the $m=2$ and $m=3$ base cases. Let $m \geq 4$ and assume the statement is true for walks of length $m-1$ and $m-2$.

If $j>k+1$ we have $h_{W}(j)=1-j<-k$ so we must have $m \leq 2 k+1$. Consider the walk $W^{\prime}=v_{1} \ldots v_{m}$ of length $m-1$. We have $h_{W^{\prime}}(i)=1-i<0$ for $2 \leq i<j$ and $h_{W^{\prime}}(i) \leq h_{W^{\prime}}(j-1)+(i-(j-1))=3+i-2 j<0$ for $j \leq i \leq m-1$. Thus the inductive hypothesis is applicable and we get $c_{1}>c_{2}>c_{m}$.

Finally consider the $j \leq k+1$ case. As the trivial $j=m$ case was already dealt with we also assume $j<m$. Clearly, $h_{W}(2)<0$ implies $j \geq 2$. We chose a $w_{0} \ldots w_{j-2}$ walk in $G$ ending at $w_{j-2}=v_{j}$ and with coloring $\left(c_{1}^{\prime}, \ldots, c_{j-2}^{\prime}\right)$ satisfying $c_{1}^{\prime}>c_{2}^{\prime}>\ldots>c_{j-2}^{\prime}>c_{j+1}$. This is possible since the edge $v_{j} v_{j+1}$ is in the $(k-1)$-shaving $G^{\prime}$ of $G$, so we can find the edge $w_{j-3} w_{j-2}$ in the $(k-2)$-shaving of $G, w_{j-4} w_{j-3}$ in the $(k-3)$-shaving, and so on. We must have $c_{1}>c_{1}^{\prime}$ as otherwise $w_{0} w_{1} \ldots w_{j-3} v_{j} v_{j-1} \ldots v_{0}$ is a $(j-1)$-fast walk and no such walk exists in $G$. Now consider the 
walk $W^{\prime}=w_{0} w_{1} \ldots w_{j-3} v_{j} v_{j+1} \ldots v_{m}$. This is a walk of length $m-2$ and satisfies $h_{W^{\prime}}(i)=1-i$ for $1 \leq i \leq j-1$ and $h_{W^{\prime}}(i)=h_{W}(i+2)$ for $j-1 \leq i \leq m-2$. All requirements of the inductive hypothesis are satisfied, so we have $c_{1}^{\prime}>c_{m}$. Thus $c_{1}>c_{1}^{\prime}>c_{m}$ as claimed.

Corollary 2.1. Let $G=(A, B, E)$ be a bipartite graph with a d edge coloring and let $k \geq 2$. There exists a $k$-flat subgraph $G^{\prime}=\left(A, B, E^{\prime}\right)$ of $G$ with $\left|E^{\prime}\right|>\frac{\log ^{(k-1)} d}{4 \cdot 240^{k-1} d}|E|-(k-1)(|A|+|B|)$.

Proof: Combine Theorem 2 nwith Lemma 2.9 and the fact that $(k-1)$ shaving keeps all but at at most $(k-1)(|A|+|B|)$ edges of $G$.

The final lemma in this section is a simple but useful observation on $k$-flat graphs. It can also be stated for longer walks with height function bounded from below, but for simplicity we restrict attention to short walks.

Lemma 2.10. Let $k \geq 1$ and let $G$ be a properly edge colored $k$-flat graph. Let $W=v_{0} \ldots v_{m}$ be a walk in $G$ of length $m \leq 2 k+1$ with coloring $\left(c_{1}, \ldots, c_{m}\right)$. If $c_{1} \geq c_{i}$ for all $1 \leq i \leq m$ then $h_{W}(i) \leq 0$ for all $1 \leq i \leq m$.

Proof: We prove the contrapositive statement. Assume $h_{W}(i)>0$ for some $1 \leq i \leq m$ and let $i_{0}$ be the smallest such index. Clearly, $i_{0} \geq 2, h_{W}\left(i_{0}\right)=1$ and for the walk $W^{\prime}=v_{i_{0}} v_{i_{0}-1} \ldots v_{0}$ we have $h_{W^{\prime}}(i)=h_{W}\left(i_{0}-i+1\right)-1<0$ for $1<i \leq i_{0}$. So by the definition of $k$-flatness we have $c_{i_{0}}>c_{1}$.

\section{Locally plane graphs}

Locally plane graphs were introduced in the paper [7] (though the name appears first in this paper). That paper gives a simple construction for 3locally plane graphs. We recall (a simplified version of) the construction as it is our starting point.

\subsection{Construction of 3-locally plane graphs in [7]}

Let $d \geq 1$ and consider the orthogonal projection of (the edge graph of) the $d$ dimensional hypercube into the plane. A suitable projection of the "middle layer" of the hypercube provides the 3-locally plane graph. Here is the construction in detail:

Let $d \geq 1$ be fixed and set $b=\lfloor d / 2\rfloor$. The bit at position $i$ in $x \in\{0,1\}^{d}$ (the $i$ th coordinate) is denoted by $x_{i}$ for $1 \leq i \leq d$. We let $A=\left\{x \in\{0,1\}^{d}\right.$ $\left.\sum_{i=1}^{d} x_{i}=b\right\}$ and $B=\left\{x \in\{0,1\}^{d} \mid \sum_{i=1}^{d} x_{i}=b+1\right\}$. The abstract graph 
underlying the geometric graph to be constructed is $G_{d}=(A, B, E)$ with $(x, y) \in E$ if $x \in A, y \in B$ and $x$ differs from $y$ in a single position. This is the middle layer of the $d$ dimensional hypercube. We define the edge coloring $\chi: E \rightarrow\{1, \ldots, d\}$ that colors an edge $e=(x, y) \in E$ by the unique position $\chi(e)=i$ with $x_{i} \neq y_{i}$. Notice that this is a proper edge coloring. The number of vertices is $n=|A|+|B|=\left(\begin{array}{l}d \\ b\end{array}\right)+\left(\begin{array}{c}d \\ b+1\end{array}\right) \leq 2^{d}$, the number of edges is $|E|=\left(\begin{array}{l}d \\ b\end{array}\right)(d-b)>n d / 4$. The average degree is greater than $d / 2 \geq \log n / 2$.

To make the abstract graph $G_{d}$ into a geometric graph we project the hypercube into the plane. We give two possible projections here. The first is more intuitive and it is closer to the actual construction in [7]. We let $a_{i}=\left(10^{i}, i \cdot 10^{i}\right)$ for $1 \leq i \leq d$ and use this vector as the projection of the edges of color $i$. We will use that among the vectors $a_{i}$ higher index means higher slope and much greater length. We identify the vertex $x \in A \cup B$ with the point $P_{x}=\sum_{i=1}^{d} x_{i} a_{i}$. The edges are represented by the straight line segment connecting their endpoints.

We give the second construction to obtain a graph where all edges are very close in length and direction. Let $0<\epsilon<10^{-d}$ be arbitrary and consider the vectors $b_{i}=\left(1+10^{i} \epsilon, \epsilon^{d+1-i}\left(1+10^{i} \epsilon\right)\right)$ and identify a point $x \in A \cup B$ with $Q_{x}=\sum_{i=1}^{d} x_{i} b_{i}$.

It is easy to verify that we get a geometric graph in both cases (i.e., the vertices are mapped to distinct points and no edge passes through a vertex that is not its endpoint). Note that edges of color $i$ are all translates of the same vector $a_{i}$ or $b_{i}$. We do not introduce separate notations for the two geometric graphs constructed this way as they will only be treated separately in the proof of Lemma 3.1, where we refer to them as the first and the second realization of $G_{d}$.

\subsection{Self-intersecting paths in $G_{d}$}

In [7] a graph very similar to $G_{d}$ was shown to be 3 -locally plane. Here we do more, we analyze all self-intersecting paths of $G_{d}$ as follows.

Lemma 3.1. Let $W$ be a walk in $G_{d}$ with coloring $\left(c_{1}, \ldots, c_{m}\right)$ satisfying $c_{1} \geq c_{m}$. Assume that $W$ and all its non-empty subwalks have a unique edge of maximal color. The first and last edges of $W$ cross in either geometric realization if and only if $m$ is even and there is an odd index $1<j<m$ satisfying $c_{1}>c_{m}>c_{j} \geq c_{i}$ for all $1<i<m$.

Proof: Let $W=v_{0} \ldots v_{m}$. Note that the first and the last edges cross if and only if $v_{0}$ and $v_{1}$ are on different sides of the line $\ell$ through $v_{m-1}$ and 
$v_{m}$ and similarly $v_{m-1}$ and $v_{m}$ are on different sides of the line $\ell^{\prime}$ through $v_{0}$ and $v_{1}$. To analyze such separations consider the projection $\pi_{i}$ to the $y$ axis parallel to edges of color $i$.

Let us consider the first realization of $G_{d}$ with the vectors $a_{i}$. We have $\pi_{i}(x, y)=y-i x$ and the projection of the vector $a_{j}$ is of length $|i-j| 10^{j}$. Thus higher colored edges map to longer intervals (except color $i$ itself). Under the projection $\pi_{c_{1}}$ the direction of the highest colored edge in the walks $v_{1} \ldots v_{m-1}$ (respectively, $v_{1} \ldots v_{m}$ ) determines which side $v_{m-1}$ (respectively $v_{m}$ ) lies of the line $\ell^{\prime}$. Indeed this highest color cannot be $c_{1}$, so the projections of the other edges will be much shorter and by the unique maximal color property we see that the walk contains at most $2^{k-1}$ edges having the $k$ th largest color, so these shorter projections cannot add up to be more than the projection of the largest edge.

We can only have $v_{m-1}$ and $v_{m}$ lying on different sides of $\ell^{\prime}$ if these edges of maximal color are distinct, thus we must have $c_{m}>c_{i}$ for all $1<i<m$. From $c_{1} \geq c_{m}$ and the unique maximal color property we have $c_{1}>c_{m}$. Taking $c_{j}$ to maximize $c_{i}$ for $1<i<m$ (this is unique again) we have $c_{1}>c_{m}>c_{j} \geq c_{i}$ for all $1<i<m$.

It is left to prove that the first and last edges of $W$ cross if and only if $m$ is even and $j$ is odd.

To prove this claim one has to use that $G_{d}$ is bipartite with vertex sets $A$ and $B$, and every edge of color $c$ is a translate of the vector $a_{c}$ with its head in $B$ and tail in $A$. Thus, the vector $v_{i-1} v_{i}$ is either $a_{c_{i}}$ or $-a_{c_{i}}$ depending on the parity of $i$. Which sides of $\ell^{\prime} v_{m-1}$ and $v_{m}$ lie is determined by the projections of the $j$ th and last edge, so they are on opposite sides if $j$ and $m$ have different parities. Similarly, the sides of $\ell$ on which $v_{0}$ and $v_{1}$ lies is detemined by the $\pi_{c_{m}}$ projection of the first and $j$ th edges, but as we have $c_{1}>c_{m}>c_{j}$ they are on different sides if 1 and $j$ has the same parity. See Figure 1 for a rough depiction of all four cases. This finishes the proof of the claim and the part of the lemma regarding the first realization of $G_{d}$.

The proof for the second realization of $G_{d}$ as a geometric graph (involving the vectors $\left.b_{i}\right)$ is slightly more complicated. We have $\pi_{i}(x, y)=y-\epsilon^{d+1-i} x$ and $\pi_{i}\left(b_{j}\right)=\epsilon^{d+1-j}\left(1+10^{j} \epsilon\right)-10^{j} \epsilon^{d+2-i}-\epsilon^{d+1-i}$. The $\epsilon^{d+1-i}$ terms alternate in sign in the projection on the edges and cancel completely for a walk of even length. For a walk of odd length a single such term remains but it is dominated by the other terms if the walk has an edge with color above $i$. If, however, no such edge exists, the remaining uncanceled $\epsilon^{d+1-i}$ term dominates the other terms in the projection. Thus, if the projection of the unique edge with the largest color of a walk has color $c>i$ or if $c<i$ but the walk has even length, then the sign of the $\pi_{i}$ projection of the edge with the 


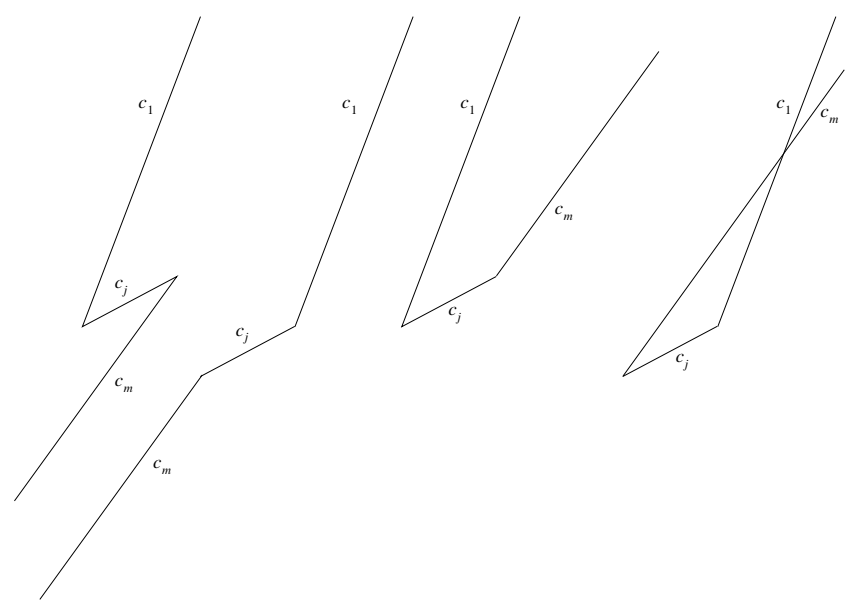

Figure 1: Rough picture of $W$ in the first realization of $G_{d}$ in the four cases according to the parities of $m$ and $j$. The scale is set to match the edge $v_{j-1} v_{j}$ of color $c_{j}$, shorter edges are approximated by zero, and only initial segments of the two longer edges are depicted.

largest color determines the sign of the projection of the entire walk. But in case $c<i$ and the walk has odd length, then the non-canceling $\epsilon^{d+1-i}$ term determines the sign.

Let the edge $v_{j-1} v_{j}$ be the one with the unique largest color in the walk $v_{1} \ldots v_{m-1}$. A case analysis of the parities of $j, m$ and whether $c_{j}>c_{1}$ or $c_{j}>c_{m}$ hold show that the first and last edges of $W$ cross if and only if $c_{m}>c_{j}, j$ is odd and $m$ is even - as claimed in the lemma.

We call $v_{m} \ldots v_{0}$ the reverse of the walk $W=v_{0} \ldots v_{m}$.

Lemma 3.2. Let $k \geq 1$ and let $G^{\prime}$ be a $k$-flat subgraph of $G_{d}$. If the length of a walk $W$ in $G^{\prime}$ does not exceed $2 k+1$, then $W$ has unique edge of largest color.

Proof: Let $W$ be a walk of length $3 \leq m \leq 2 k+1$ in $G^{\prime}$ with coloring $\left(c_{1}, \ldots, c_{m}\right)$ and assume the largest color is not unique. We may assume $c_{1}=c_{m}>c_{i}$ holds for all $1<i<m$, otherwise one can take a suitable subwalk of $W$. By Lemma 2.10 we have $h_{W}(m) \leq 0$. Consider $W^{\prime}$ the reverse of the walk $W$. Clearly, $h_{W^{\prime}}(m)=-h_{W}(m)$, but also by Lemma 2.10 we have $h_{W^{\prime}}(m) \leq 0$. So we must have $h_{W}(m)=0$ and $m$ must be odd.

The contradiction that proves the statement of the lemma comes from the simple observation that between two consecutive appearances of a color 
in any walk of $G_{d}$ there always are an even number of edges, so $m$ must be even. To see this recall that $G_{d}$ is bipartite with sides $A$ and $B$ where $A$ consists of the $0-1$ sequences of length $d$ with $\lfloor d / 2\rfloor$ ones, while the $0-1$ sequences in $B$ contain one more ones. The $A$ end of an edge of color $c$ has 0 at position $c$, while the $B$ end of this edge has 1 there. Along edges of other colors bit $c$ does not change. Thus if a walk traverses an edge of color $c$ from $A$ to $B$ say, then along the walk bit $c$ remains 1 until the next time the walk traverses an edge of color $c$ and this has to be from $B$ to $A$.

We note that Lemma 3.2 immediately implies that the girth of a $k$-flat subgraph of $G_{d}$ is at least $2 k+2$. This estimate can be improved by observing that any cycle in $G_{d}$ has an even number of edges of any color (one has to flip a bit even times to get beck to the original state). In particular any cycle has at least two occurrences of the largest color. These edges break the cycle into two paths sharing two edges. Both path has to be of length at least $2 k+2$, the length of the cycle is at least $4 k+2$.

As every properly edge colored graph is 1 -flat the $k=1$ case of the next lemma establishes that $G_{d}$ is 3-locally plane.

Lemma 3.3. For any $k \geq 1$ any $k$-flat subgraph of $G_{d}$ is $(2 k+1)$-locally plane in either realization.

Proof: Let $G^{\prime}$ be a $k$-flat subgraph of $G_{d}$. We need to show that no walk (or path) $W$ of length $m \leq 2 k+1$ is self-intersecting. It is clearly enough to show that the first and the last edges of $W$ do not cross and we may assume that the color of the first edge is not smaller than that of the last edge (otherwise simply consider the same walk reversed). By Lemma 3.2 $W$ and all its subwalks have a unique edge of maximal color, so Lemma 3.1 applies. It is enough to show that the coloring $\left(c_{1}, \ldots, c_{m}\right)$ of $W$ does not satisfy the conditions of Lemma 3.1. Assume the contrary. So $m$ is even, and there is an odd index $j$ such that $c_{1}>c_{m}>c_{j} \geq c_{i}$ for all $1<i<m$.

Consider the walk $W_{1}=v_{m} v_{m-1} \ldots v_{j-1}$ of length $m-j+1$. Its coloring is $\left(c_{m}, \ldots, c_{j}\right)$ and $c_{m}$ is its largest color. By Lemma 2.10 $h_{W_{1}}(m-j+1) \leq 0$. In fact, as $m-j+1$ is even $h_{W_{1}}(m-j+1)$ is odd, so we have $h_{W_{1}}(m-j+1) \leq$ -1 .

Consider the walk $W_{2}=v_{j} v_{j-1} \ldots v_{1}$ of length $j-1$ and its coloring $\left(c_{j}, \ldots, c_{2}\right)$. The largest color in $W_{2}$ is $c_{j}$, so we have $h_{W_{2}}(j-1) \leq 0$ by Lemma 2.10. And again by parity considerations $h_{W_{2}}(j-1) \leq-1$.

It is easy to see that $h_{W}(m)=-1-h_{W_{2}}(j-1)-h_{W_{1}}(m-j+1)$. So we have $h_{W}(m) \geq-1+1+1=1$ contradicting Lemma 2.10. The contradiction proves Lemma 3.3 . 
Theorem 3. For any fixed $k>0$ and large enough $n$ there exists a $(2 k+1)$ locally plane graphs on $n$ vertices having at least $\left(\frac{\log ^{(k)} n}{240^{k}}-k\right) n$ edges. Given two arbitrary disks in the plane, one can further assume that all edges of these graph connect a vertex inside one disk to one inside the other disk.

Proof: Simply combine the results of Corollary 2.1 and Lemma 3.3. If $n$ is not the size of the vertex set of $G_{d}$ for any $d$, add isolated vertices to the largest $G_{d}$ with fewer than $n$ vertices. Use the second realization of $G_{d}$ with a small enough $\epsilon>0$ to obtain a geometric graph with all edges connecting two small disks and apply a homothety and a rotation to get to the desired disks.

\section{Discussion on optimality of thinning}

The maximum number of edges of a 3-locally plane graph on $n$ vertices is $\Theta(n \log n)$ as proved in [7]. The lower bound is reproduced here by the $k=1$ case of Theorem 3, which is therefore tight. The upper bound of [7] extends to $x$-monotone topological graphs, i.e., when the edges are represented by curves with the property that every line parallel to the $y$ axis intersects an edge at most once. Without this artificial assumption on $x$-monotonicity only much weaker upper bounds are known. For higher values of $k$ we do not have tight results even if the edges are straight line segments as considered in this paper. While the number of edges in a $k$-locally plane graph constructed here deteriorates very rapidly with the increase of $k$, the upper bound hardly changes. In fact the only known upper bound better than $O(n \log n)$ is for 5-locally plane graphs: they have $O(n \log n / \log \log n)$ edges as shown in [7]. Slightly better bounds are known for geometric (or $x$-monotone topological) graphs with the additional condition that a vertical line intersects every edge. If such a graph is $(2 k)$-locally plane for $k \geq 2$, then it has $O\left(n \log ^{1 / k} n\right)$ edges. The first realization of $G_{d}$ does not satisfy this condition, but the second one does. Still, the lower and upper bounds for this restricted problem are far apart: for 4-locally plane graphs with a cutting line the upper bound on the number of edges is $O(n \sqrt{\log n})$ while the construction gives $\Omega(n \log \log n)$.

Although we cannot establish that the locally plane graphs constructed are optimal we can prove that the thinning procedure we use is optimal within a constant factor. It follows that any 4-locally plane subgraph of either realization of $G_{d}$ has $O(n \log \log n)$ edges. This optimality result below

refers to a single step of the thinning procedure. It would be interesting to 
establish a strong upper bound on the number of edges of a $k$-flat graph for $k \geq 3$.

Let us mention here that the thinning procedures described in this paper found application in the extremal theory of 0-1 matrices, see [12], and there the result is shown to be optimal within a constant factor. Consider an $n$ by $n$ 0-1 matrix that has no 2 by 3 submatrix of either of the following two forms:

$$
\left(\begin{array}{lll}
1 & 1 & * \\
1 & * & 1
\end{array}\right), \quad\left(\begin{array}{lll}
1 & * & 1 \\
* & 1 & 1
\end{array}\right),
$$

where the $*$ can represent any entry. The maximal number of 1 entries in such a matrix is $\Theta(n \log \log n)$ as proved in [12]. The construction proving the lower bound is based on lexicographic thinning.

The following lemma shows that the number of edges in the subgraphs claimed in Lemma 2.1 and Theorem 1 are optimal in a very strong sense: no properly edge colored graph with significantly more edges than the ones guaranteed by the above results can avoid heavy paths (slow or fast walks, respectively).

Lemma 4.1. Let $G=(A, B, E)$ be a bipartite graph with proper edge coloring given by $\chi: E \rightarrow\{1, \ldots, d\}$.

a) If $G$ does not have a heavy path then $|E| \leq 2 \sqrt{d|A||B|} \leq(|A|+|B|) \sqrt{d}$.

b) If $G$ does not have a slow walk then $|E| \leq(|A|+|B|)(\log d+2)$.

c) If $G$ does not have a fast walk then $|E| \leq 2(|A|+|B|)(\log d+2)$.

Proof: For any vertex $z \in A \cup B$ denote by $m(z)=\max (\chi(e))$, where the maximum is for edges $e$ incident to $z$. For an edge $e=(x, y) \in E$ we define its weight to be $w(e)=m(x)-\chi(e)$, while its $B$-weight is $w_{B}(e)=m(y)-\chi(e)$. Clearly, both $w(e)$ and $w_{B}(e)$ are integers in $[0, d-1]$.

To prove part a) of the lemma assume $G$ does not contain a heavy path. We set a threshold parameter $t=\lfloor\sqrt{d|A| /|B|}\rfloor$ and call an edge $e$ B-light if $w_{B}(e)<t$, otherwise $e$ is $B$-heavy.

All the edges incident to a vertex $y \in B$ have different colors, thus they also have different $B$-weights, so at most $t$ of them can be $B$-light. The total number of $B$-light edges is at most $|B| t \leq \sqrt{d|A||B|}$.

Now consider two edges $e_{1}=\left(x, y_{1}\right)$ and $e_{2}=\left(x, y_{2}\right)$ incident to vertex $x \in A$. Assume $\chi\left(e_{2}\right) \leq \chi\left(e_{1}\right)$. If $w_{B}\left(e_{2}\right)>0$ we can extend the path formed by these two edges with the edge $e_{3}$ incident to $y_{2}$ having maximum color $\chi\left(e_{3}\right)=m\left(y_{2}\right)$. Clearly, $\chi\left(e_{3}\right)=\chi\left(e_{2}\right)+w_{B}\left(e_{2}\right)$ and the resulting path 
is heavy unless $\chi\left(e_{2}\right)>\chi\left(e_{1}\right)+w\left(e_{2}\right)$. Therefore, the number of $B$-heavy edges incident to $x$ is at most $d /(t+1)$. The total number of $B$-heavy edges is at most $|A| d /(t+1) \leq \sqrt{d|A||B|}$.

For the total number of edges we have $|E| \leq 2 \sqrt{d|A||B|} \leq(|A|+|B|) \sqrt{d}$.

For parts b) and c) of the lemma consider an edge $e=(x, y) \in E$. If $w(e)=0$ we call the edge $e$ maximal. Clearly, there are at most $|A|$ maximal edges. If $e$ is not maximal we define $n(e)$ to be the "next larger colored edge at $x$ ", i.e., $n(e)$ is the edge in $E$ having minimal color $\chi(n(e))$ among edges incident to $x$ and satisfying $\chi(n(e))>\chi(e)$. We define the gap of $e$ to be $g(e)=\chi(n(e))-\chi(e)$. Clearly, $0<g(e) \leq w(e)$. We call the edge $e$ heavy if $w(e)>2 g(e)$, otherwise $e$ is light. Recall, that for maximal edges $n(e)$ and $g(e)$ are not defined and maximal edges are neither light nor heavy.

Let $e_{1}$ and $e_{2}$ be distinct edges in $E$ incident to a vertex $x \in A$. If $\chi\left(e_{1}\right)<\chi\left(e_{2}\right)$ then $w\left(e_{1}\right) \geq w\left(e_{2}\right)+g\left(e_{1}\right)$. If $e_{1}$ is light, $w\left(e_{1}\right) \geq 2 w\left(e_{2}\right)$ follows, therefore at most $\lceil\log d\rceil$ light edges are incident to $x \in A$. Thus the total number of light edges in $E$ is at most $\lceil\log d\rceil|A|$.

For part b) of the lemma assume $G$ does not contain a slow walk. Let $e_{2}=\left(x_{2}, y\right)$ and $e_{3}=\left(x_{3}, y\right)$ be distinct non-maximal edges in $E$ and assume $\chi\left(e_{2}\right)<\chi\left(e_{3}\right)$. Let $e_{1}=n\left(e_{2}\right)$ and let $e_{4}$ be the maximal edge incident to $x_{3}$ in $G$. We have $\chi\left(e_{1}\right)=\chi\left(e_{2}\right)+g\left(e_{2}\right)$ and $\chi\left(e_{4}\right)=\chi\left(e_{3}\right)+w\left(e_{3}\right)$. The edges $e_{1}, e_{2}, e_{3}$, and $e_{4}$ cannot form a slow walk. As $\chi\left(e_{1}\right)>\chi\left(e_{2}\right)<\chi\left(e_{3}\right)<\chi\left(e_{4}\right)$ we must have $\chi\left(e_{4}\right)<\chi\left(e_{1}\right)$. This implies $g\left(e_{2}\right)>w\left(e_{3}\right)$, and if $e_{2}$ is heavy $w\left(e_{2}\right)>2 w\left(e_{3}\right)$. Therefore, at most $\lceil\log d\rceil$ heavy edges can be incident to $y \in B$. The total number of heavy edges in $G$ is at most $(\lceil\log d\rceil|B|$.

For the total number of edges we add the bound obtained for light, heavy, and maximal edges and get $|E| \leq(|A|+|B|)\lceil\log d\rceil+|A|$.

Finally for part c) of the lemma we assume $G$ does not contain a fast walk. Let $E_{1}$ consist of the edges $(x, y) \in E$ for which $m(x) \geq m(y)$. Assume without loss of generality that $\left|E_{1}\right| \geq|E| / 2$. If this is not the case consider the same graph with its sides switched. We use here that the definition of a fast walk and the claimed bound on the number of edges are both symmetric in the color classes.

As in the previous case consider two non-maximal edges $e_{2}=\left(x_{2}, y\right)$ and $e_{3}=\left(x_{3}, y\right)$ in $E_{1}$ with $\chi\left(e_{2}\right)<\chi\left(e_{3}\right)$. Let $e_{1}$ be the maximal edge incident to $x_{2}$ and let $e_{4}=n\left(e_{3}\right)$. As $\chi\left(e_{2}\right)<\chi\left(e_{3}\right)<\chi\left(e_{4}\right)$ but $G$ does not contain a fast walk we must have $\chi\left(e_{1}\right)<\chi\left(e_{4}\right)$. As $e_{2} \in E_{1}$ we must also have 
$\chi\left(e_{1}\right)=m\left(x_{2}\right) \geq m(y) \geq \chi\left(e_{3}\right)$. If $e_{3}$ is heavy we also have

$$
\begin{aligned}
\chi\left(e_{3}\right)+w\left(e_{3}\right)-m(y) & >\chi\left(e_{3}\right)+2 g\left(e_{3}\right)-m(y) \\
& \geq 2\left(\chi\left(e_{3}\right)+g\left(e_{3}\right)-m(y)\right) \\
& =2\left(\chi\left(e_{4}\right)-m(y)\right) \\
& >2\left(\chi\left(e_{1}\right)-m(y)\right) \\
& =2\left(\chi\left(e_{2}\right)+w\left(e_{2}\right)-m(y)\right) .
\end{aligned}
$$

For all the heavy edges $e \in E_{1}$ incident to $y \in B$ the values $\chi(e)+w(e)-m(y)$ increase strictly more than by a factor of 2 . As these values are integers from $[0, d-1]$, there are at most $\lceil\log d\rceil$ heavy edges in $E_{1}$ incident to $y$. The total number of heavy edges in $E_{1}$ is at most $\lceil\log d\rceil|B|$.

For the total number of edges in $E_{1}$ we sum our bound on heavy edges in $E_{1}$ and the bounds on the light and maximal edges in $E$. We obtain $\left|E_{1}\right| \leq$ $(|A|+|B|)\lceil\log d\rceil+|A|$. Finally we get $|E| \leq 2(|A|+|B|)\lceil\log d\rceil+2|A|$.

\section{References}

[1] E. Ackerman, On the maximum number of edges in topological graphs with no four pairwise crossing edges, Discrete and Computational Geometry 41 (2009) (3), 365-375.

[2] P. Agarwal, B. Aronov, J. Pach, R. Pollack, M. Sharir, Quasi-planar graphs have a linear number of edges, Combinatorica 17 (1997), 1-9.

[3] S. Avital, H. Hanani, Graphs, Gilyonot Lematematika 3 (1966), 2-8 (in Hebrew).

[4] A. Marcus, G. Tardos, Intersection reverse sequences and geometric applications, Journal of Combinatorial Theory, Series A 113 (2006), 675-691.

[5] J. Pach, Geometric graph theory, in: Surveys in Combinatorics, 1999, J. Lamb, D. Preece (eds.), London Mathematical Society Lecture Notes 267, cambridge University Press, Cambridge, 1999, pp. 167-200.

[6] J. Pach, Geometric graph theory, in: Handbook of Discrete and Computational Geometry, J. Goodman, J. O'Rourke (eds.), CRC Press, Boca Raton, FL (Chapter 10).

[7] J. Pach, R. Pinchasi, G. Tardos, G. Tóth, Geometric graphs with no self-intersecting path of length three, European Journal of Combinatorics 25 (2004) (6), 793-811. 
[8] J. Pach, R. Radoičić, G. Tóth, Relaxing planarity for topological graphs, in: Discrete and Computational Geometry (J. Akiyama, M. Kano, eds.), Lecture Notes in Computer Science 2866, Springer-Verlag, Berlin, 2003, pp. 221-232.

[9] J. Pach. G. Tóth, Graph drawn with few crossings per edge, Combinatorica 17 (1997), 427-439.

[10] R. Pinchasi, R. Radoičić, On the number of edges in geometric graphs with no self-intersecting cycle of length 4, in: Towards a Theory of Geometric Graphs, 233-243, Contemporary Mathematics 342, American Mathematical Society, Providence, RI, 2004.

[11] P. Valtr, Graph drawing with no $k$ pairwise crossing edges, in Graph Drawing (Rome, 1997), 205-218, Lecture Notes in Comput. Sci., 1353, Springer, Berlin, 1997.

[12] G. Tardos, On 0-1 matrices and small excluded submatrices, Journal of Combinatorial Theory, Series A 111 (2005), 266-288.

[13] G. Tardos, G. Tóth, Crossing stars in topological graphs, SIAM Journal on Discrete Mathematics 21 (2007), 737-749. 